\title{
Pilot Study of a Prototype Integrated Diabetes Management System and the Relationship of Use to Diabetes Management Behaviors and HbA1c Among Type 2 Diabetes Patients
}

Connor Devoe ${ }^{1}$, BS; Nils Fischer ${ }^{1}$, MPH; Tim Hale ${ }^{2}$, PhD; Neda Derakhshani ${ }^{1}$; Mursal Atif ${ }^{1}$; Harriett Gabbidon ${ }^{1}$; Enrico Cagliero $^{3}$, MD; Marie McDonnell ${ }^{4,5}$, MD; Kamal Jethwani ${ }^{1,6,7}$, MD, MPH

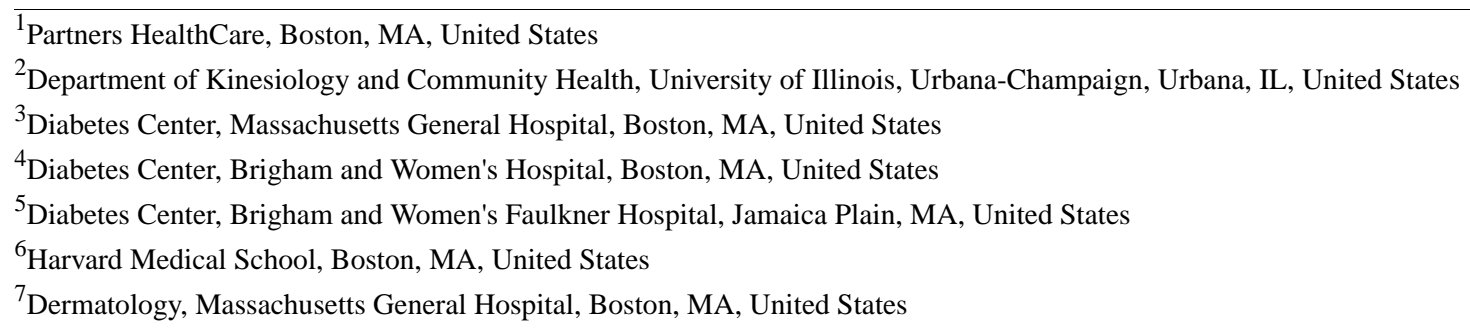

\section{Corresponding Author:}

Connor Devoe, BS

Partners HealthCare

25 New Chardon Street

Boston, MA

United States

Phone: 6037481896

Email: $\underline{\text { cdevoe@bu.edu }}$

\section{Abstract}

Background: Type 2 diabetes (T2D) is the seventh leading cause of death (2017) in the United States, and by 2030 it is estimated that it will affect 439 million globally. Effective glycemic control can be challenging for patients. A tool to guide patients' in their self-management behaviors and share this data with their physician may improve insulin adherence leading to lower $\mathrm{HbA}_{1 \mathrm{c}}$. We examined an integrated diabetes management (IDM) system that utilizes a Bluetooth-enabled insulin event capture device, a Bluetooth-enabled glucometer, and an Android smartphone app. IDM data can be viewed by clinicians in the electronic medical record (EMR).

Objective: The primary aim of this study is to describe how app use is related to insulin adherence, blood glucose measurements, meal snapshots, and step count. Secondarily, we assessed the impact on $\mathrm{HbA}_{1 \mathrm{c}}$ levels over a 3- and 6-month period.

Methods: Thirty-five participants were enrolled from Boston-area hospitals in this single-arm pilot study. Use of the IDM system was defined as the number of days per week participants logged into the app and moved past the home screen. Three app use groups were created: low app use (0.33-2.46 days per week), medium app use (2.54-5.08 days per week), and high app use (>5.4 days per week). Adherence to insulin, blood glucose measurements, and meal snapshots were defined as a ratio of actual weekly events recorded by participants' app use divided by their physician's recommendation. Step count was defined as the total weekly steps for each participant. Daily app-generated data on app use and indicators of diabetes management were collected. $\mathrm{HbA}_{1 \mathrm{c}}$ levels were assessed via blood test at enrollment, 3-months, and 6-months. Using a hierarchical linear mixed model, we examined changes in outcome measures while accounting for random intercepts and slopes to control for variation in individual outcomes over the study.

Results: Overall app use (average unique days using the app per week) declined from 6.19 days to 3.00 days (at 1 and 24 weeks, respectively). Participants with high app use had significant improvement in bolus and basal insulin adherence per week $(0.009$ $P=.041$ [95\% CI 0.0004 to 0.018 ] and $0.016 P<.001$ [95\% CI 0.0079 to 0.023 ], respectively), but participants had no significant improvements in blood glucose and meal snapshot adherence or absolute step count. $\mathrm{HbA}_{1 \mathrm{c}}$ significantly decreased per week (coefficient -0.025 [95\% CI -0.044 to -0.007$], P=.007$ ) with an overall change of 0.6 . Participants with high app use significantly improved their $\mathrm{HbA}_{1 \mathrm{c}}$ per week $(-0.037 P=.016$ [ -0.066 to -0.0067$\left.]\right)$ compared to participants with medium and low app use, yielding a total improvement of 0.88 over 24 weeks. 
Conclusions: Results show that bolus and basal insulin may have increased with higher app use. HbA $1 \mathrm{c}$ significantly improved over the course of the study, along with significantly greater improvement in $\mathrm{HbA}_{1 \mathrm{c}}$ among participants with higher app use compared to participants in the middle or low app use groups. This study is not designed or intended to evaluate efficacy but provides results to guide the future design and development of this prototype IDM system.

(iproc 2019;5(1):e16298) doi: 10.2196/16298

\section{KEYWORDS}

health behavior; type 2 diabetes mellitus; digital health; mobile app; IDM; patient engagement

Edited by J Brown; this is a non-peer-reviewed article. Submitted 17.09.19; accepted 17.09.19; published 02.10.19.

Please cite as:

Devoe C, Fischer N, Hale T, Derakhshani N, Atif M, Gabbidon H, Cagliero E, McDonnell M, Jethwani K

Pilot Study of a Prototype Integrated Diabetes Management System and the Relationship of Use to Diabetes Management Behaviors and HbAlc Among Type 2 Diabetes Patients iproc 2019;5(1):e16298

URL: http://www.iproc.org/2019/1/e16298/

doi: $10.2196 / 16298$

PMID:

(C) Connor Devoe, Nils Fischer, Tim Hale, Neda Derakhshani, Mursal Atif, Harriett Gabbidon, Enrico Cagliero, Marie McDonnell, Kamal Jethwani. Originally published in Iproceedings (http://www.iproc.org), 02.10.2019 This is an open-access article distributed under the terms of the Creative Commons Attribution License (https://creativecommons.org/licenses/by/4.0/), which permits unrestricted use, distribution, and reproduction in any medium, provided the original work, first published in Iproceedings, is properly cited. The complete bibliographic information, a link to the original publication on http://www.iproc.org/, as well as this copyright and license information must be included. 